\title{
OPTIMIZATION OF DRILLING PROCESS PARAMETERS VIA TAGUCHI, TOPSIS AND RSA TECHNIQUES
}

\begin{abstract}
Carbon Fiber Reinforced Polymer (CFRP) is the most preferred composite material due to its high strength, high modulus, corrosion resistance and rigidity and which has wide applications in aerospace engineering, automobile sector, sports instrumentation, light trucks, airframes. This paper is an attempt to carry out drilling experiments as per Taguchi's $\mathrm{L}_{27}\left(3^{13}\right)$ orthogonal array on CFRP under dry condition with three different drill bit type (HSS, TiAlN and TiN). In this research work Response Surface Analysis (RSA) is used to correlate the effect of process parameters (cutting speed and feed rate) on thrust force, torque, vibration and surface roughness. This paper also focuses on determining the optimum combination of input process parameter and the drill bit type that produces quality holes in CFRP composite laminate using Multi-objective Taguchi technique and TOPSIS. The percentage of contribution, influence of process parameters and adequacy of the second order regression model is carried out by analysis of variance (ANOVA). The results of experimental investigation demonstrates that feed rate is the pre-dominate factor which affects the response variables.
\end{abstract}

Keywords: CFRP, Taguchi, TOPSIS, RSA, Vibration, Optimization

\section{Introduction}

Carbon Fiber Reinforced Polymer (CFRP) is well-known for their extraordinarily high strength, high modulus, corrosion resistance and rigidity. Carbon fibers reinforced with epoxy resin usually find very good application because of their strength and stiffness combination. The CFRPs are superior to steel and glass fiber reinforced polymer (GFRP) with respect to specific tensile strength and specific elastic modulus. CFRPs with their positive characteristics are mostly used in applications like aerospace engineering, automobile sector, sports instrumentation, industrial sector, shafts etc. Joining of composite structures is an unavoidable process in many applications; hence the quality of the machined hole plays a vital role on it. During drilling of CFRPs several problems like fuzzing, fiber-pullout, fiber breakage, delamination, debonding of fiber/matrix etc., occurs due to its anisotropic and non-homogeneity nature of structures. Conventional method of drilling remains at top priority in lieu of its mechanical/thermal properties and also the cost and quality of the manufactured goods.

Arul et al. [1] proposed a new technique by inducing vibration in the direction of feed, there by reduces thrust force and improves the quality of drilled holes. Ramesh et al. [2] have worked on multiple performance characteristics of drilling process parameters using Grey Relational Analysis (GRA), TOPSIS and Response Surface Methodology (RSM). The adequacy of the developed model is validated by means of
ANOVA. Quality holes can be drilled on bi-directional CFRP composites using TiN-SC tool. Since TiN-SC exhibits lower thrust force, surface roughness of the drilled holes improves considerably [3]. Kurt et al. [4] performed an experimental investigation with three different drilling tools on hole quality and surface roughness in drilling of Al 2024 in dry condition. Taguchi technique is successfully used to optimize the machining process parameters. Grilo et al. [5] studied the influence of drill geometry and optimize the process parameters in drilling of CFRP using three different drill bits with the aim of reducing the delamination and increasing the production rate. Tsao and Hocheng [6] conducted an experimental survey of thrust force and surface roughness models in drilling of CFRP. Correlation between the machining process parameter and the responses are obtained by neural network and regression analysis. Sheth and George [7] investigated the effect of machining process parameters during drilling of Wrought Cast Steel Grade B (WCB) on cylindricity and perpendicularity. Taguchi $\mathrm{L}_{27}\left(3^{13}\right)$ orthogonal array is used for the experimental design and the significance of process parameters is evaluated by ANOVA. Nouari et al. [8] analyzed the influence of input process parameters in drilling aluminium alloy on tool life and hole quality. They have recommended using reduced web thickness, increasing the helix and point angle to optimal drill geometry. Feed rate and drill type are the significant factors which affect thrust force while feed rate and cutting speed contributes more to the total variation of torque [9]. Neseli [10] investigated the effect of

* VJCET, VAZHAKULAM, DEPARTMENT OF MECHANICAL ENGINEERING, KERALA, INDIA
** NIT, TRICHY, DEPARTMENT OF PRODUCTION ENGINEERING, TAMILNADU, INDIA

* Corresponding author: shunmugesh@gmail.com 
machining process parameters in drilling of AISI 1040 steel with the aim of reducing thrust force and torque. The responses are minimized at higher cutting speed and low feed rate. The major damages encountered in drilling are at the entry and exit of the holes and surface roughness [11]. The indirect measurement of these damage are through thrust force and torque whereas the direct measurement are through delamination factor and surface roughness. Jayabal and Natarajan [12] studied the effect of machining parameters in drilling of coir fiber reinforced composites on thrust force, torque and tool wear. Their results indicate that the minimum thrust force is at higher cutting speed and lower feed rate. Dewagan et al. [13] applied Fuzzy-TOPSIS coupled with sensitivity analysis to optimize the EDM process parameters with multiple performance characteristics. Gok [14] developed a new approach to optimize the turning process parameters using Fuzzy-TOPSIS, Grey Relational Analysis (GRA) and Response Surface Analysis (RSA). Madhavan et al. [15] conducted drilling simulation on CFRP to predict the force and observed that the fiber orientation angle is the most influencing process parameter. Zhang et al. [16] optimized turbodrill blades design using response surface methodology (RSM) and reported that the efficiency of the optimized design increases the performances by $10 \%$. Bobbili et al. [17] conducted experiments to study the effect of WEDM parameters on multiple performance characteristics, - MRR and Ra and also optimized the input process parameters.

The objective of the current work is to study the effect of input process parameters (cutting speed, feed rate and drill bit type) in drilling of CFRP composite laminate on the experimental results of thrust force, torque, vibration and surface roughness. Finally optimum condition to obtain minimum thrust force, torque, vibration and surface roughness when drilling $6 \mathrm{~mm}$ diameter hole on a $8 \mathrm{~mm}$ thick laminate are found out using Multi-objective Taguchi technique, TOPSIS and RSA. In addition significance and contribution of input process parameters are identified using ANOVA.

\section{Materials and methods}

In this paper CFRP manufactured by hand layup and auto clave was chosen as the work piece material for conducting drilling experiments. The work piece used for the experiments is CFRP (T300 Bi-directional Carbon Fibre/Epoxy matrix with a volume fraction of $60 \%$ ). The carbon fiber used in the material is of PAN-based and the average thickness of the fabric is $0.25 \mathrm{~mm}$. The size of the specimen used was $150 \times 15 \times 8 \mathrm{~mm}$. The experiments were carried out using BFW Ltd BMV 40T20 $\mathrm{CNC}$ vertical milling machine. The cutting tools used for the dry drilling are HSS drill (Miranda Tools India Ltd), Kennametal Solid Carbide Drill (TiAlN Black Coated-KC7325 Grade) and WIDIA Solid Carbide Drill (TiN Golden coated - WU25PD Grade). The drilling tools of $6 \mathrm{~mm}$ diameter and 2 flutes are used for all the experimental trials. The surface roughness value $\mathrm{Ra}$ was measured by using Mitutoyo SJ-210 surface roughness tester and for better results, average of three replicates was found out. The Kistler multicomponent dynamometer 9257B was used to measure the three orthogonal components of cutting forces i.e., $\mathrm{Fx}, \mathrm{Fy}$ and $\mathrm{Fz}$ for all the trial experiments. The dynamometer consists of multi-component measuring system and multi-charge amplifier channels. These channels convert the charge signals from the dynamometer into output signals. A piezoelectric accelerometer with a sampling rate of 25600 samples/second was used as vibration sensor (Dytran make 3055B) and it was mounted on the top of the workpiece. The signals from the pickup were fed to a signal conditioner and a vibration indicator. Tool vibration, particularly in the radial direction, is known to have a deleterious effect on the machined surface texture. During this study, 3 controllable process parameters: cutting speed, feed rate and drill bit type are elite and varied in 3 completely different levels as shown in Table 1. The schematic experimental setup is shown in Fig. 1.

TABLE 1

Machining parameters and their levels

\begin{tabular}{|c|c|c|c|c|}
\hline \hline \multirow{2}{*}{ Process parameters } & \multirow{2}{*}{ Symbol } & \multicolumn{3}{|c|}{ Levels } \\
\cline { 3 - 5 } & & $\mathbf{1}$ & $\mathbf{2}$ & $\mathbf{3}$ \\
\hline Cutting speed, $\mathrm{m} / \mathrm{min}$ & $v$ & 30 & 40 & 50 \\
\hline Feed rate, $\mathrm{mm} / \mathrm{rev}$ & $f$ & 0.025 & 0.05 & 0.1 \\
\hline Drill bit type & $d$ & HSS & TiAlN & TiN \\
\hline
\end{tabular}

\section{Result and discussions}

\subsection{Response Surface Analysis (RSA)}

The second order regression model for thrust force, torque, vibration and surface roughness were obtained and are represented in Table 2. These regression models are used to predict the experimental results of thrust force, torque, vibration and surface roughness at certain conditions of process parameter.

The predicted values of $F_{o}, T_{o}, V i b$ and $R a$ were obtained from Table 2 are compared with experimental results. It is clear and evident that the predicted values are in good agreement with the experimental results as shown in Fig. 2.

\subsection{Multi-objective Taguchi Membership Function (MOTMF)}

In this section, initially the experimental results are analyzed and evaluated individually. Later the MOTMF is used to for the multiple performance characteristics. The effect of machining process parameters on drilling of CFRP was analyzed using responses surface analysis. The experimental results of thrust force, torque, vibration and surface roughness are shown in Table 3. Now using the MOTMF approach, the multi-objective function is converted into single objective function. The higher value of $\mathrm{S} / \mathrm{N}$ ratio indicates better performance. The experimental responses, normalized data, membership function, $\mathrm{MOF}$ and $\mathrm{S} / \mathrm{N}$ 
Regression model for thrust force, torque, vibration and surface roughness

\begin{tabular}{|c|c|c|}
\hline Regression model & $R^{2}$ & Pred. $R^{2}$ \\
\hline $\begin{aligned} F_{O}= & 100.554-0.0388889 v+454.698 f-21.6361 d-0.0113889 v^{\wedge} 2 \\
& -1345.19 f^{\wedge} 2+5.94444 d^{\wedge} 2+1.69524 v f-0.0741667 v d-20.1429 f d\end{aligned}$ & 94.3 & 87.10 \\
\hline $\begin{aligned} T o= & 74.0537-1.83250 v+286.286 f-14.4917 d+0.0183333 v^{\wedge} 2 \\
& -1828.15 f^{\wedge} 2+3.26667 d^{\wedge} 2+0.70 v f+0.0108333 v d+6.52381 f d\end{aligned}$ & 97.6 & 93.15 \\
\hline $\begin{aligned} V i= & 0.0357296+0.000324 v+0.497556 f-0.0187278 d-7.555 e^{\wedge}(-6) v^{\wedge} 2 \\
& -2.8 f^{\wedge} 2+0.004194 d^{\wedge} 2-0.00105714 v f+4.58333 e^{\wedge}(-5) v d+0.00780952 f d\end{aligned}$ & 94.2 & 85.72 \\
\hline $\begin{aligned} R a= & 8.74222-0.186972 v+23.5603 f-0.640778 d+0.00191944 v^{\wedge} 2-123.348 f^{\wedge} 2 \\
& +0.13811 d^{\wedge} 2+0.0570476 v f+0.000841667 v d-0.413333 f d\end{aligned}$ & 98.9 & 97.24 \\
\hline
\end{tabular}

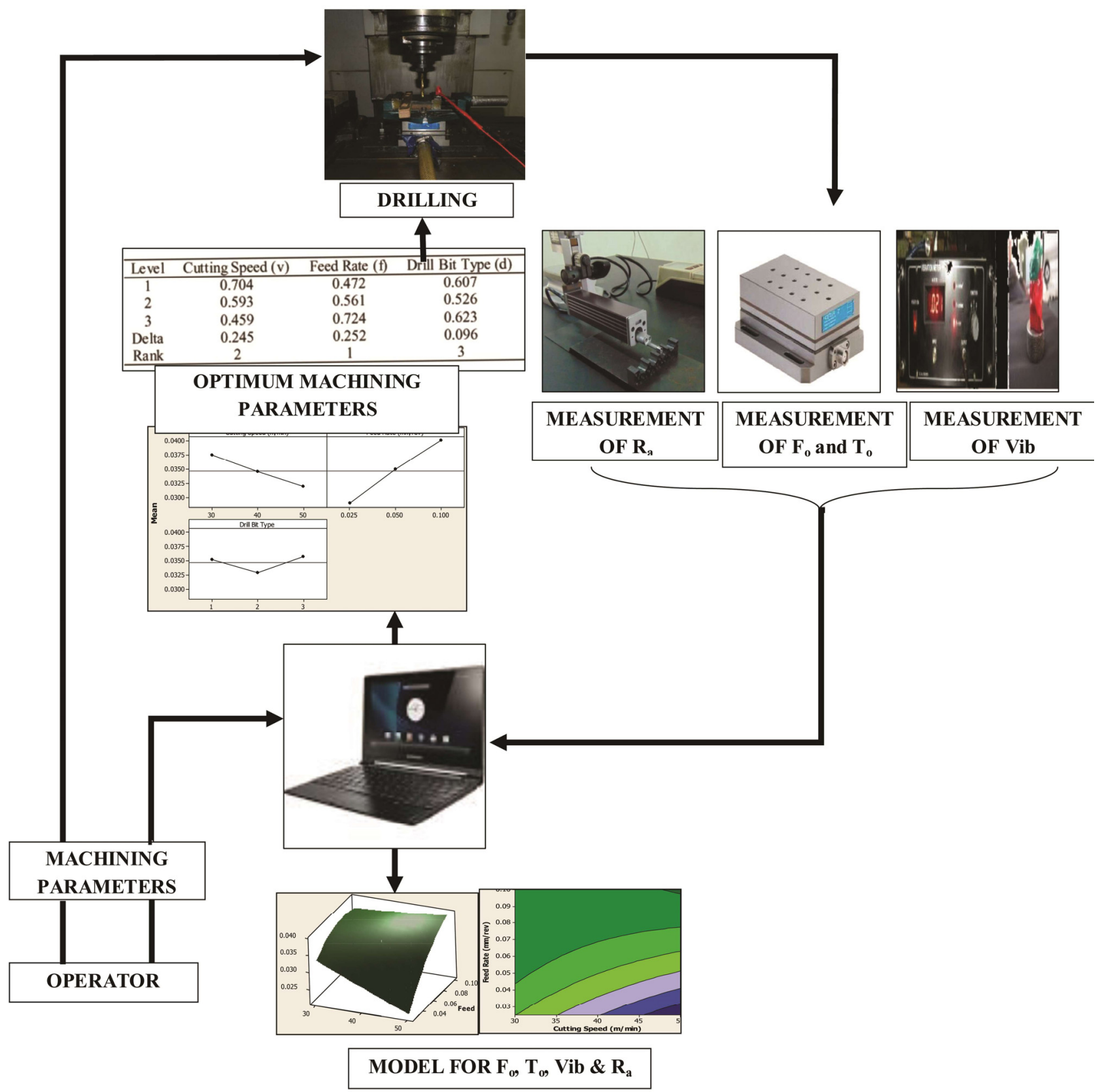

Fig. 1. Experimental setup 

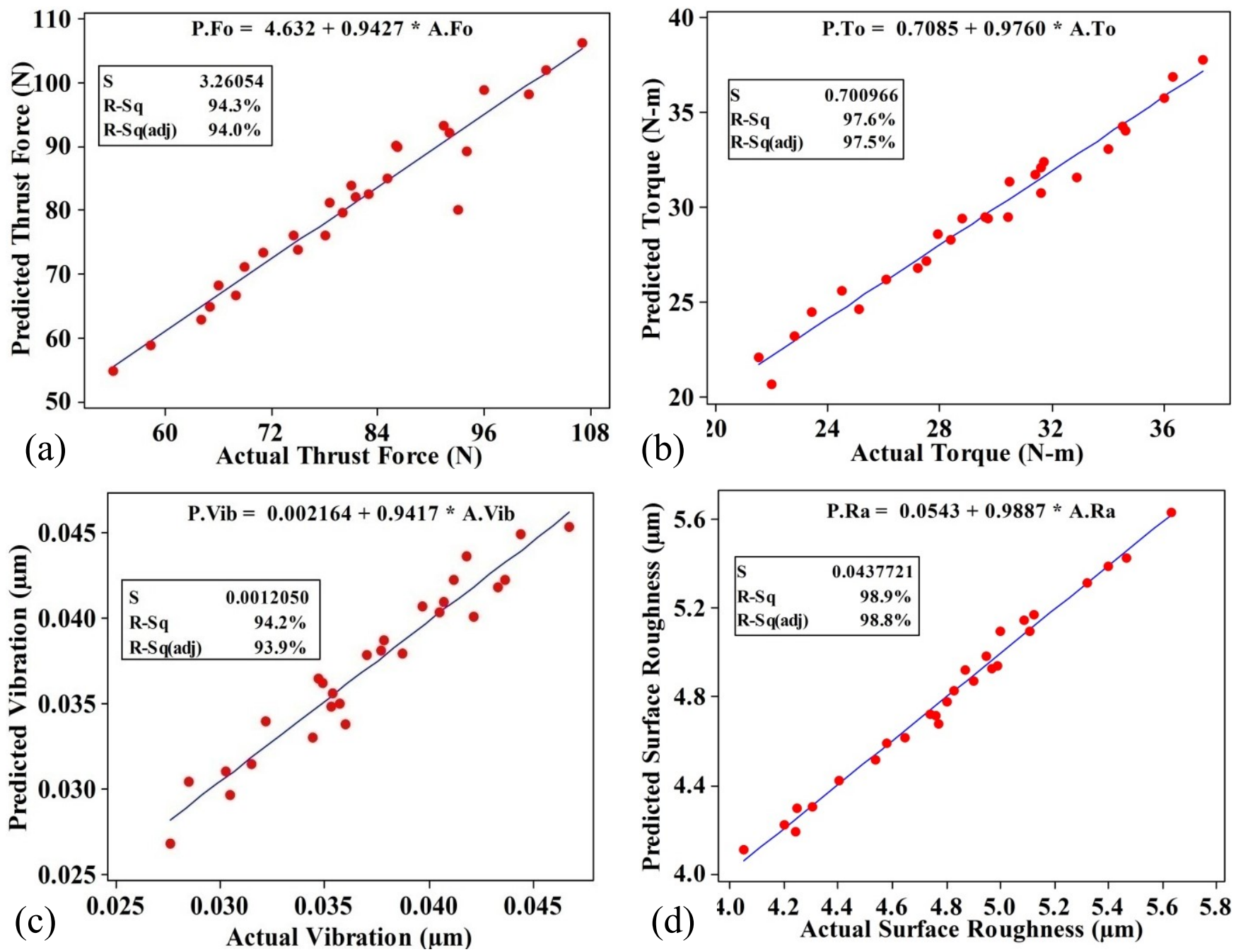

Fig. 2. Comparison plots of actual and predicted values of a) Thrust force b) Torque c) Vibration and d) Surface roughness

TABLE 3

Experimental design, responses, normalized data, membership function, $\mathrm{MOF}$ and $\mathrm{S} / \mathrm{N}$ ratio

\begin{tabular}{|c|c|c|c|c|c|c|c|c|c|c|c|c|c|c|c|c|c|}
\hline \multirow[b]{2}{*}{$\begin{array}{c}\text { Exp } \\
\text { No }\end{array}$} & \multicolumn{3}{|c|}{ Control factor } & \multicolumn{4}{|c|}{ Responses } & \multicolumn{4}{|c|}{ Normalized data } & \multicolumn{4}{|c|}{ Membership function } & \multirow[b]{2}{*}{ MOF } & \multirow{2}{*}{$\begin{array}{c}\text { S/N } \\
\text { Ratio }\end{array}$} \\
\hline & $v$ & $f$ & $d$ & $\begin{array}{c}R_{a} \\
(\mu \mathrm{m})\end{array}$ & $\begin{array}{l}F_{o} \\
(\mathrm{~N})\end{array}$ & $\begin{array}{c}T_{o} \\
(\mathbf{N m})\end{array}$ & $\begin{array}{c}V_{i} \\
(\mu \mathrm{m})\end{array}$ & $\begin{array}{c}R_{a} \\
(\mu \mathrm{m})\end{array}$ & $\begin{array}{l}F_{o} \\
(\mathbf{N}) \\
\end{array}$ & $\begin{array}{c}T_{o} \\
(\mathbf{N m})\end{array}$ & $\begin{array}{c}V_{i} \\
(\mu \mathrm{m})\end{array}$ & $\begin{array}{c}R_{a} \\
(\mu \mathrm{m})\end{array}$ & $\begin{array}{l}F_{o} \\
(\mathbf{N}) \\
\end{array}$ & $\begin{array}{c}T_{o} \\
(\mathbf{N m})\end{array}$ & $\begin{array}{c}V_{i} \\
(\mu \mathrm{m})\end{array}$ & & \\
\hline 1 & 2 & 3 & 4 & 5 & 6 & 7 & 8 & 9 & 10 & 11 & 12 & 13 & 14 & 15 & 16 & 17 & 18 \\
\hline 1 & 30 & 0.025 & HSS & .971 & 83 & 30.5 & 0.035 & 0.574 & 0.542 & 0.559 & 0.418 & 0.396 & 0.429 & 0.411 & 0.560 & 0.585 & 4.661 \\
\hline 2 & 30 & 0.025 & TiAlN & 4.76 & 78 & 27.5 & 0.030 & 0.454 & 0.458 & 0.390 & 0.177 & 0.522 & 0.518 & 0.590 & 0.813 & 0.315 & 10.032 \\
\hline 3 & 30 & 0.025 & TiN & 4.8 & 78.5 & 30.4 & 0.035 & 0.477 & 0.466 & 0.554 & 0.413 & 0.498 & 0.509 & 0.417 & 0.565 & 0.485 & 6.285 \\
\hline 4 & 30 & 0.05 & HSS & 5.32 & 92 & 36 & 0.041 & 0.773 & 0.695 & 0.871 & 0.691 & 0.187 & 0.268 & 0.083 & 0.273 & 1.219 & -1.718 \\
\hline 5 & 30 & 0.05 & TiAlN & 5 & 85 & 31.4 & 0.037 & 0.590 & 0.576 & 0.610 & 0.493 & 0.378 & 0.393 & 0.357 & 0.481 & 0.682 & 3.320 \\
\hline 6 & 30 & 0.05 & TiN & 5.09 & 86.2 & 34.5 & 0.043 & 0.642 & 0.597 & 0.786 & 0.790 & 0.325 & 0.372 & 0.173 & 0.169 & 1.057 & -0.485 \\
\hline 7 & 30 & 0.1 & HSS & 5.632 & 107 & 37.4 & 0.044 & 0.950 & 0.950 & 0.950 & 0.842 & 0.000 & 0.000 & 0.000 & 0.114 & 1.798 & -5.095 \\
\hline 8 & 30 & 0.1 & TiAlN & 5.4 & 101 & 34.6 & 0.040 & 0.818 & 0.848 & 0.792 & 0.667 & 0.139 & 0.107 & 0.167 & 0.298 & 1.295 & -2.244 \\
\hline 9 & 30 & 0.1 & TiN & 5.47 & 103 & 36.3 & 0.046 & 0.858 & 0.882 & 0.888 & 0.950 & 0.097 & 0.071 & 0.066 & 0.000 & 1.687 & -4.540 \\
\hline 10 & 40 & 0.025 & HSS & 4.404 & 75 & 26.1 & 0.036 & 0.251 & 0.407 & 0.310 & 0.446 & 0.735 & 0.572 & 0.673 & 0.531 & 0.276 & 11.195 \\
\hline 11 & 40 & 0.025 & TiAlN & 4.197 & 68 & 21.5 & 0.030 & 0.134 & 0.288 & 0.050 & 0.187 & 0.859 & 0.697 & 0.947 & 0.804 & 0.073 & 22.778 \\
\hline 12 & 40 & 0.025 & TiN & 4.245 & 69 & 23.4 & 0.032 & 0.161 & 0.305 & 0.158 & 0.267 & 0.831 & 0.679 & 0.834 & 0.719 & 0.113 & 18.937 \\
\hline 13 & 40 & 0.05 & HSS & 4.83 & 81 & 31.6 & 0.042 & 0.494 & 0.508 & 0.622 & 0.733 & 0.480 & 0.465 & 0.346 & 0.228 & 0.751 & 2.490 \\
\hline 14 & 40 & 0.05 & TiAlN & 4.647 & 74.5 & 27.2 & 0.034 & 0.390 & 0.398 & 0.373 & 0.394 & 0.590 & 0.581 & 0.608 & 0.585 & 0.318 & 9.949 \\
\hline 15 & 40 & 0.05 & $\mathrm{TiN}$ & 4.771 & 93 & 29.7 & 0.039 & 0.460 & 0.712 & 0.514 & 0.620 & 0.516 & 0.250 & 0.459 & 0.347 & 0.720 & 2.853 \\
\hline 16 & 40 & 0.1 & HSS & 5.125 & 96 & 34 & 0.043 & 0.662 & 0.763 & 0.758 & 0.804 & 0.304 & 0.197 & 0.203 & 0.154 & 1.179 & -1.431 \\
\hline 17 & 40 & 0.1 & TiAlN & 4.99 & 86 & 29.6 & 0.037 & 0.585 & 0.593 & 0.508 & 0.531 & 0.384 & 0.375 & 0.465 & 0.441 & 0.650 & 3.747 \\
\hline
\end{tabular}




\begin{tabular}{|c|c|c|c|c|c|c|c|c|c|c|c|c|c|c|c|c|c|}
\hline 1 & 2 & 3 & 4 & 5 & 6 & 7 & 8 & 9 & 10 & 11 & 12 & 13 & 14 & 15 & 16 & 17 & 18 \\
\hline 18 & 40 & 0.1 & TiN & 4.95 & 91.5 & 31.7 & 0.041 & 0.562 & 0.687 & 0.627 & 0.719 & 0.408 & 0.277 & 0.340 & 0.243 & 0.894 & 0.975 \\
\hline 19 & 50 & 0.025 & HSS & 4.302 & 64 & 25.1 & 0.028 & 0.193 & 0.220 & 0.254 & 0.092 & 0.796 & 0.769 & 0.733 & 0.903 & 0.083 & 21.566 \\
\hline 20 & 50 & 0.025 & TiAlN & 4.05 & 54 & 22 & 0.027 & 0.050 & 0.050 & 0.078 & 0.050 & 0.947 & 0.947 & 0.918 & 0.947 & 0.007 & 42.884 \\
\hline 21 & 50 & 0.025 & TiN & 4.24 & 58.4 & 22.8 & 0.031 & 0.158 & 0.125 & 0.124 & 0.234 & 0.834 & 0.869 & 0.870 & 0.754 & 0.058 & 24.710 \\
\hline 22 & 50 & 0.05 & HSS & 4.74 & 71 & 28.8 & 0.034 & 0.443 & 0.339 & 0.463 & 0.385 & 0.534 & 0.643 & 0.512 & 0.595 & 0.354 & 9.015 \\
\hline 23 & 50 & 0.05 & TiAlN & 4.538 & 65 & 24.5 & 0.034 & 0.328 & 0.237 & 0.220 & 0.370 & 0.655 & 0.751 & 0.769 & 0.610 & 0.184 & 14.720 \\
\hline 24 & 50 & 0.05 & TiN & 4.58 & 66 & 28.4 & 0.038 & 0.352 & 0.254 & 0.441 & 0.573 & 0.630 & 0.733 & 0.536 & 0.397 & 0.374 & 8.545 \\
\hline 25 & 50 & 0.1 & HSS & 5.11 & 94 & 31.6 & 0.037 & 0.653 & 0.729 & 0.622 & 0.526 & 0.313 & 0.232 & 0.346 & 0.446 & 0.853 & 1.378 \\
\hline 26 & 50 & 0.1 & TiAlN & 4.9 & 80 & 27.9 & 0.035 & 0.534 & 0.492 & 0.412 & 0.432 & 0.438 & 0.483 & 0.566 & 0.546 & 0.465 & 6.660 \\
\hline 27 & 50 & 0.1 & $\mathrm{TiN}$ & 4.87 & 81.4 & 32.9 & 0.040 & 0.516 & 0.515 & 0.695 & 0.658 & 0.456 & 0.458 & 0.268 & 0.308 & 0.762 & 2.357 \\
\hline
\end{tabular}

ratio are presented in Table 3. From the Table it is evident that the experiment number 20 has attained the maximum value of $\mathrm{S} / \mathrm{N}$ ratio amoung the 27 number of experiment and the optimum condition to achieve the multiple performance characteristics $(\mathrm{S} / \mathrm{N}$ ratio $=48.074$, cutting speed $=50 \mathrm{~m} / \mathrm{min}$, feed rate $=0.025 \mathrm{~mm} /$ rev and TiAlN drill bit type).

The mean effect Table and graph of $\mathrm{S} / \mathrm{N}$ ratio are given in Table 4 and Fig. 3, respectively. In MOTMF, maximum value of $\mathrm{S} / \mathrm{N}$ ratio indicates better performance. Thus the optimum level of process parameter is the one with maximum $\mathrm{S} / \mathrm{N}$ ratio value. Therefore, to achieve minimum value of thrust force, torque, vibration and surface roughness, simultaneously, the optimum combination of machining parameters obtained are $v_{3}$ (cutting speed, $50 \mathrm{~m} / \mathrm{min}$ ), $f_{1}$ (feed rate, $0.025 \mathrm{~mm} / \mathrm{rev}$ ) and $d_{2}$ (drill bit type, TiAlN).

TABLE 4

Mean effect TABLE of S/N ratio

\begin{tabular}{|c|c|c|c|}
\hline \hline Level & Cutting speed & Feed rate & Drill bit type \\
\hline 1 & 1.135 & $\mathbf{1 8 . 1 1 7}$ & 4.673 \\
\hline 2 & 7.944 & 5.410 & $\mathbf{1 2 . 4 2 7}$ \\
\hline 3 & $\mathbf{1 4 . 6 4 8}$ & 0.201 & 6.626 \\
\hline Max - Min & 13.513 & 17.916 & 1.953 \\
\hline Rank & 2 & 1 & 3 \\
\hline
\end{tabular}

\subsection{TOPSIS study}

Initially all the four experimental results of thrust force, torque, vibration and surface roughness are normalized. In the current study all the four responses is assigned equal weightage, hence the weight of thrust force, torque, vibration and surface roughness are taken as 0.25 . Once the weights are assigned, the weighted normalized decision matrix is calculated. From the weighted normalized decision matrix, the positive and negative ideal solutions are calculated. The positive and negative ideal solutions of thrust force, torque, vibration and surface roughness are said to be $u_{F o}^{+}=0.5727, u_{F o}^{-}=0.2890$, $u_{T o}^{+}=0.3311, u_{T o}^{-}=0.1903, u_{V i b}^{+}=0.0116, u_{V i b}^{-}=0.0068$, $u_{R a}^{+}=0.1235$ and $u_{R a}^{-}=0.0888$. Finally, the comparative closeness of distinct alternative is calculated, and is presented in Table 5.

From the Table 5, it is evident that the experiment number 20 was the better performer. The order of the experimental run obtained by TOPSIS was given by $20>21>19>23>11>12$ $>24>22>10>14>2>26>3>13>1>27>17>5>6>15$ $>18>25>4>16>8>9>7$. The higher value of comparative closeness value indicates better performance. From the Table 5, it is evident that the experiment number 20 has attained the maximum value of comparative closeness amoung the 27 number of experiment and the optimum condition to achieve the multiple

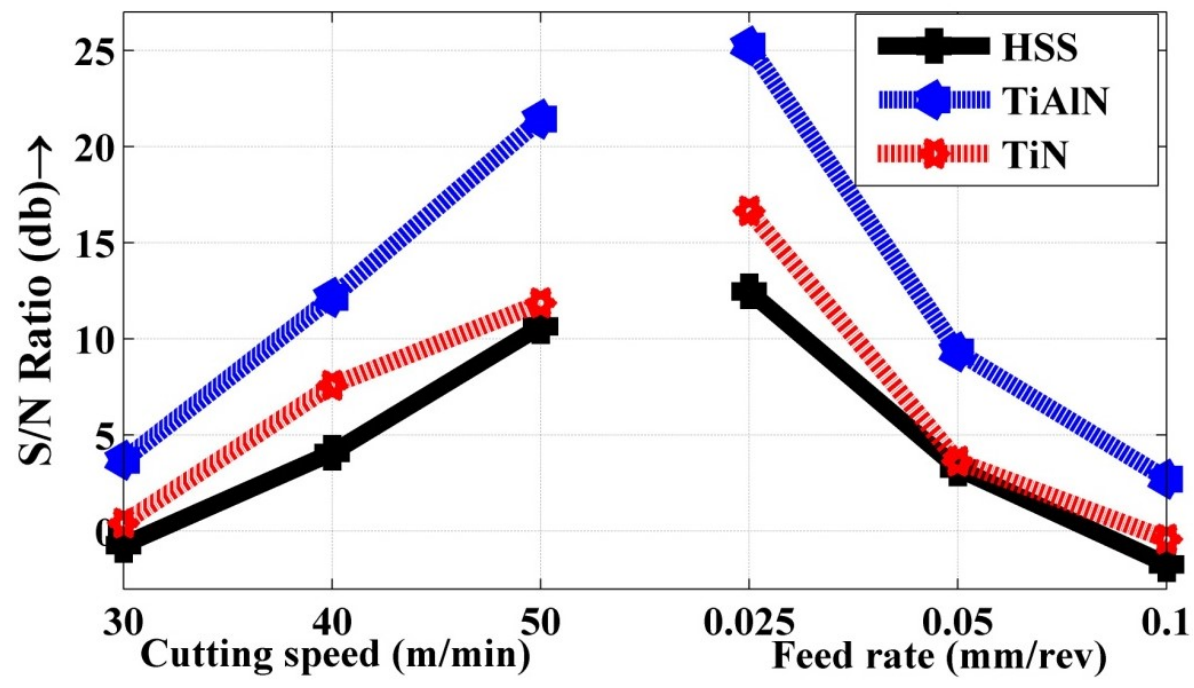

Fig. 3. Mean effect plot of $\mathrm{S} / \mathrm{N}$ ratio 
TOPSIS (normalized \& weighted normalized data, separation measures and comparative nearness)

\begin{tabular}{|c|c|c|c|c|c|c|c|c|c|c|c|}
\hline \multirow{2}{*}{$\begin{array}{c}\text { Exp } \\
\text { No }\end{array}$} & \multicolumn{4}{|c|}{$\begin{array}{c}\text { Normalized } \\
\text { data }\end{array}$} & \multicolumn{4}{|c|}{$\begin{array}{c}\text { Weighted } \\
\text { normalized data }\end{array}$} & \multicolumn{2}{|c|}{$\begin{array}{c}\text { Separation } \\
\text { measures }\end{array}$} & \multirow{2}{*}{$\begin{array}{c}\text { Comparative } \\
\text { nearness }\end{array}$} \\
\hline & $R_{a}(\mu \mathrm{m})$ & $F_{o}(\mathrm{~N})$ & $T_{o}(\mathrm{Nm})$ & $V_{i}(\mu \mathrm{m})$ & $R_{a}(\mu \mathrm{m})$ & $F_{o}(\mathrm{~N})$ & $T_{o}(\mathrm{Nm})$ & $V_{i}(\mu \mathrm{m})$ & $S_{I}^{+}$ & $S_{I}^{-}$ & \\
\hline 1 & 0.436 & 1.777 & 1.080 & 0.035 & 0.109 & 0.444 & 0.270 & 0.009 & 0.1756 & 0.1430 & 0.44878 \\
\hline 2 & 0.418 & 1.670 & 0.974 & 0.030 & 0.104 & 0.418 & 0.243 & 0.008 & 0.1398 & 0.1793 & 0.56179 \\
\hline 3 & 0.421 & 1.681 & 1.077 & 0.035 & 0.105 & 0.420 & 0.269 & 0.009 & 0.1538 & 0.1656 & 0.51847 \\
\hline 4 & 0.467 & 1.970 & 1.275 & 0.041 & 0.117 & 0.492 & 0.319 & 0.010 & 0.2421 & 0.0815 & 0.25190 \\
\hline 5 & 0.439 & 1.820 & 1.112 & 0.037 & 0.110 & 0.455 & 0.278 & 0.009 & 0.1888 & 0.1299 & 0.40765 \\
\hline 6 & 0.447 & 1.846 & 1.222 & 0.043 & 0.112 & 0.461 & 0.305 & 0.011 & 0.2085 & 0.1148 & 0.35520 \\
\hline 7 & 0.494 & 2.291 & 1.324 & 0.044 & 0.124 & 0.573 & 0.331 & 0.011 & 0.3186 & 0.0005 & 0.00180 \\
\hline 8 & 0.474 & 2.162 & 1.225 & 0.041 & 0.118 & 0.541 & 0.306 & 0.010 & 0.2786 & 0.0409 & 0.12805 \\
\hline 9 & 0.480 & 2.205 & 1.285 & 0.047 & 0.120 & 0.551 & 0.321 & 0.012 & 0.2948 & 0.0237 & 0.07465 \\
\hline 10 & 0.386 & 1.606 & 0.924 & 0.036 & 0.097 & 0.401 & 0.231 & 0.009 & 0.1198 & 0.2002 & 0.62557 \\
\hline 11 & 0.368 & 1.456 & 0.761 & 0.030 & 0.092 & 0.364 & 0.190 & 0.008 & 0.0750 & 0.2537 & 0.77186 \\
\hline 12 & 0.372 & 1.477 & 0.829 & 0.032 & 0.093 & 0.369 & 0.207 & 0.008 & 0.0821 & 0.2401 & 0.74511 \\
\hline 13 & 0.424 & 1.734 & 1.119 & 0.042 & 0.106 & 0.434 & 0.280 & 0.011 & 0.1708 & 0.1493 & 0.46649 \\
\hline 14 & 0.408 & 1.595 & 0.963 & 0.035 & 0.102 & 0.399 & 0.241 & 0.009 & 0.1215 & 0.1972 & 0.61878 \\
\hline 15 & 0.419 & 1.991 & 1.052 & 0.040 & 0.105 & 0.498 & 0.263 & 0.010 & 0.2216 & 0.1030 & 0.31745 \\
\hline 16 & 0.450 & 2.055 & 1.204 & 0.044 & 0.112 & 0.514 & 0.301 & 0.011 & 0.2517 & 0.0670 & 0.21037 \\
\hline 17 & 0.438 & 1.841 & 1.048 & 0.038 & 0.109 & 0.460 & 0.262 & 0.009 & 0.18685 & 0.13269 & 0.41526 \\
\hline 18 & 0.434 & 1.959 & 1.123 & 0.042 & 0.109 & 0.490 & 0.281 & 0.010 & 0.2210 & 0.0982 & 0.30776 \\
\hline 19 & 0.377 & 1.370 & 0.889 & 0.028 & 0.094 & 0.343 & 0.222 & 0.007 & 0.0625 & 0.2563 & 0.80387 \\
\hline 20 & 0.355 & 1.156 & 0.779 & 0.028 & 0.089 & 0.289 & 0.195 & 0.007 & 0.0044 & 0.3166 & 0.98621 \\
\hline 21 & 0.372 & 1.250 & 0.807 & 0.031 & 0.093 & 0.313 & 0.202 & 0.008 & 0.0265 & 0.2921 & 0.91665 \\
\hline 22 & 0.416 & 1.520 & 1.020 & 0.035 & 0.104 & 0.380 & 0.255 & 0.009 & 0.1126 & 0.2081 & 0.64884 \\
\hline 23 & 0.398 & 1.392 & 0.868 & 0.034 & 0.100 & 0.348 & 0.217 & 0.009 & 0.0654 & 0.2533 & 0.79456 \\
\hline 24 & 0.402 & 1.413 & 1.006 & 0.039 & 0.100 & 0.353 & 0.251 & 0.010 & 0.0894 & 0.2346 & 0.72399 \\
\hline 25 & 0.448 & 2.013 & 1.119 & 0.038 & 0.112 & 0.503 & 0.280 & 0.009 & 0.2332 & 0.0872 & 0.27230 \\
\hline 26 & 0.430 & 1.713 & 0.988 & 0.036 & 0.107 & 0.428 & 0.247 & 0.009 & 0.1514 & 0.168 & 0.52595 \\
\hline 27 & 0.427 & 1.743 & 1.165 & 0.040 & 0.107 & 0.436 & 0.291 & 0.010 & 0.1789 & 0.1436 & 0.44532 \\
\hline
\end{tabular}

performance characteristics (cutting speed $=50 \mathrm{~m} / \mathrm{min}$, feed rate $=0.025 \mathrm{~mm} / \mathrm{rev}$ and TiAlN drill bit type). Thus, the results of TOPSIS technique are in conformity with the multi-objective Taguchi technique.

\subsection{ANOVA}

Analysis of Variance (ANOVA) is used to validate the regression models and the results are presented in Table 6-9.

TABLE 6

Analysis of Variance for Thrust Force

\begin{tabular}{|c|c|c|c|c|c|c|}
\hline Source & DF & Seq. SS & Adj. SS & Adj. MS & $\mathbf{F}$ & $\mathbf{P}$ \\
\hline Regression & 9 & 4635.65 & 4635.65 & 515.07 & 31.06 & 0.000 \\
\hline Linear & 3 & 4380.76 & 4315.26 & 1438.42 & 86.73 & 0.000 \\
\hline$v$ & 1 & 1798.00 & 1741.58 & 1741.58 & 105.01 & 0.000 \\
\hline$f$ & 1 & 2510.76 & 2496.89 & 2496.89 & 150.55 & 0.000 \\
\hline$d$ & 1 & 72.00 & 76.80 & 76.80 & 4.63 & 0.046 \\
\hline Square & 3 & 236.16 & 236.16 & 78.72 & 4.75 & 0.014 \\
\hline$v \times v$ & 1 & 7.78 & 7.78 & 7.78 & 0.47 & 0.503 \\
\hline$f \times f$ & 1 & 16.36 & 16.36 & 16.36 & 0.99 & 0.335 \\
\hline$d \times d$ & 1 & 212.02 & 212.02 & 212.02 & 12.78 & 0.002 \\
\hline Interaction & 3 & 18.73 & 18.73 & 6.24 & 0.38 & 0.771 \\
\hline$v \times f$ & 1 & 5.03 & 5.03 & 5.03 & 0.30 & 0.589 \\
\hline$v \times d$ & 1 & 6.60 & 6.60 & 6.60 & 0.40 & 0.537 \\
\hline$f \times d$ & 1 & 7.10 & 7.10 & 7.10 & 0.43 & 0.522 \\
\hline Residual Error & 17 & 281.94 & 281.94 & 16.58 & & \\
\hline Total & 26 & 4917.59 & & & & \\
\hline
\end{tabular}


TABLE 7

Analysis of Variance for Torque

\begin{tabular}{|c|c|c|c|c|c|c|}
\hline \hline Source & DF & Seq. SS & Adj. SS & Adj. MS & F & P \\
\hline Regression & 9 & 512.054 & 512.054 & 56.895 & 76.85 & 0.000 \\
\hline Linear & 3 & 395.904 & 412.791 & 137.597 & 185.86 & 0.000 \\
\hline$v$ & 1 & 165.620 & 159.600 & 159.600 & 215.58 & 0.000 \\
\hline$f$ & 1 & 223.562 & 247.161 & 247.161 & 333.85 & 0.000 \\
\hline$d$ & 1 & 6.722 & 6.030 & 6.030 & 8.14 & 0.011 \\
\hline Square & 3 & 114.407 & 114.407 & 38.136 & 51.51 & 0.000 \\
\hline$v \times v$ & 1 & 20.167 & 20.167 & 20.167 & 27.24 & 0.000 \\
\hline$f \times f$ & 1 & 30.213 & 30.213 & 30.213 & 40.81 & 0.000 \\
\hline$d \times d$ & 1 & 64.027 & 64.027 & 64.027 & 86.48 & 0.000 \\
\hline Interaction & 3 & 1.743 & 1.743 & 0.581 & 0.78 & 0.519 \\
\hline$v \times f$ & 1 & 0.857 & 0.857 & 0.857 & 1.16 & 0.297 \\
\hline$v \times d$ & 1 & 0.141 & 0.141 & 0.141 & 0.19 & 0.668 \\
\hline$f \times d$ & 1 & 0.745 & 0.745 & 0.745 & 1.01 & 0.330 \\
\hline Residual Error & 17 & 12.586 & 12.586 & 0.740 & & \\
\hline Total & 26 & 524.640 & & & & \\
\hline
\end{tabular}

TABLE 8

Analysis of Variance for Vibration

\begin{tabular}{|c|c|c|c|c|c|c|}
\hline \hline Source & DF & Seq. SS & Adj. SS & Adj. MS & F & P \\
\hline Regression & 9 & 0.000623 & 0.000623 & 0.000069 & 30.51 & 0.000 \\
\hline Linear & 3 & 0.000437 & 0.000487 & 0.000162 & 71.56 & 0.000 \\
\hline$v$ & 1 & 0.000113 & 0.000114 & 0.000114 & 50.47 & 0.000 \\
\hline$f$ & 1 & 0.000323 & 0.000370 & 0.000370 & 163.13 & 0.000 \\
\hline$d$ & 1 & 0.000002 & 0.000002 & 0.000002 & 1.08 & 0.314 \\
\hline Square & 3 & 0.000180 & 0.000180 & 0.000060 & 26.44 & 0.000 \\
\hline$v \times v$ & 1 & 0.000003 & 0.000003 & 0.000003 & 1.51 & 0.236 \\
\hline$f \times f$ & 1 & 0.000071 & 0.000071 & 0.000071 & 31.25 & 0.000 \\
\hline$d \times d$ & 1 & 0.000106 & 0.000106 & 0.000106 & 46.55 & 0.000 \\
\hline Interaction & 3 & 0.000006 & 0.000006 & 0.000002 & 0.81 & 0.503 \\
\hline$v \times f$ & 1 & 0.000002 & 0.000002 & 0.000002 & 0.86 & 0.366 \\
\hline$v \times d$ & 1 & 0.000003 & 0.000003 & 0.000003 & 1.11 & 0.306 \\
\hline$f \times d$ & 1 & 0.000001 & 0.000001 & 0.000001 & 0.47 & 0.502 \\
\hline Residual Error & 17 & 0.000039 & 0.000039 & 0.000002 & & \\
\hline Total & 26 & 0.000661 & & & & \\
\hline
\end{tabular}

TABLE 9

Analysis of Variance for Surface Roughness

\begin{tabular}{|c|c|c|c|c|c|c|}
\hline \hline Source & DF & Seq. SS & Adj. SS & Adj. MS & F & P \\
\hline Regression & 9 & 4.24751 & 4.24751 & 0.47195 & 165.61 & 0.000 \\
\hline Linear & 3 & 3.76493 & 3.84907 & 1.28302 & 450.22 & 0.000 \\
\hline$v$ & 1 & 1.45238 & 1.40312 & 1.40312 & 492.36 & 0.000 \\
\hline$f$ & 1 & 2.20084 & 2.33136 & 2.33136 & 818.08 & 0.000 \\
\hline$d$ & 1 & 0.11171 & 0.11460 & 0.11460 & 40.21 & 0.000 \\
\hline Square & 3 & 0.47305 & 0.47305 & 0.15768 & 55.33 & 0.000 \\
\hline$v \times v$ & 1 & 0.22106 & 0.22106 & 0.22106 & 77.57 & 0.000 \\
\hline$f \times f$ & 1 & 0.13754 & 0.13754 & 0.13754 & 48.26 & 0.000 \\
\hline$d \times d$ & 1 & 0.11445 & 0.11445 & 0.11445 & 40.16 & 0.000 \\
\hline Interaction & 3 & 0.00954 & 0.00954 & 0.00318 & 1.12 & 0.370 \\
\hline$v \times f$ & 1 & 0.00570 & 0.00570 & 0.00570 & 2.00 & 0.176 \\
\hline$v \times d$ & 1 & 0.00085 & 0.00085 & 0.00085 & 0.30 & 0.592 \\
\hline$f \times d$ & 1 & 0.00299 & 0.00299 & 0.00299 & 1.05 & 0.320 \\
\hline Residual Error & 17 & 0.04845 & 0.04845 & 0.00285 & & \\
\hline Total & 26 & 4.29596 & & & & \\
\hline
\end{tabular}



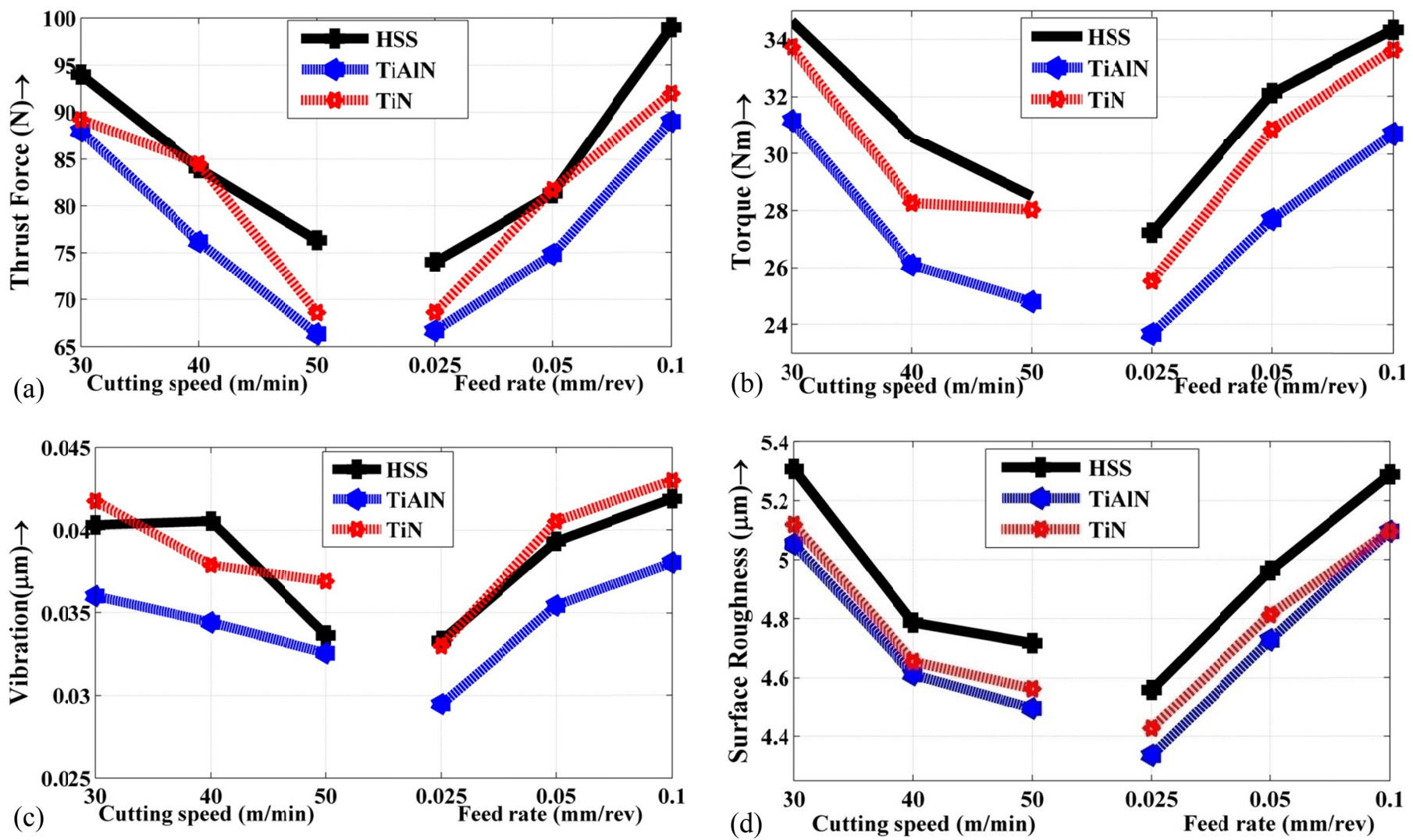

Fig. 4. Parametric influence of machining parameters on a) Thrust force, b) Torque c) Vibration and d) Surface roughness

The effect of input process parameters on the experimental results are studied by means of ANOVA. From the ANOVA Table of all the four responses it is evident that the cutting speed, feed rate and drill bit type have significant effect on the responses as the "p" value of the models are less than 0.05 . Also the adequacy of the model was validated by co-efficient of determination $\left(R^{2}\right)$ and the results are shown in Table 2 . The response surface methodology (RSM) performed by the statistical software (MINITAB 16) was used for mathematical modelling of the experimental results. The second order regression model of thrust force, torque, vibration and surface roughness are developed by the correlation of input process parameters using the RSM technique.

The main effect plot and ANOVA results of thrust force, torque, vibration and surface roughness are shown in Fig. 4 and Table 6-9 respectively. According to the experimental data, an increase in $66.6 \%$ of cutting speed results in decreasing the magnitude of thrust force by $31.82 \%$ but increasing the feed rate by $75 \%$ increases the thrust force by $32.83 \%$. Feed rate is found to be the principal factor which affects the thrust force as it contributes $54.50 \%$ of total variation, followed by cutting speed $(38.95 \%)$ and drill bit type $(5.30 \%)$. Minimum value of thrust force is observed to be $54 \mathrm{~N}$ at $50 \mathrm{~m} / \mathrm{min}, 0.025 \mathrm{~mm} / \mathrm{rev}$ and with TiAlN drill bit type.

Feed rate contributes $51.39 \%$ to the total variation of torque, followed by cutting speed (36.72\%) and drill bit type $(5.78 \%)$. From the experimental data, it is observed that $75 \%$ increase in feed rate increases torque by $34.8 \%$ of total variation.
Mean while, an increase of $66.6 \%$ in cutting speed decreases the torque by $24 \%$ of total variation. Minimum value of torque is observed to be $22 \mathrm{Nm}$ at $50 \mathrm{~m} / \mathrm{min}, 0.025 \mathrm{~mm} / \mathrm{rev}$ and with TiAlN drill bit type.

According to the experimental results, an increase in $66.6 \%$ of cutting speed results in decreasing the magnitude of vibration by $10.77 \%$ but increasing the feed rate by $75 \%$ increases the vibration by $31.03 \%$. Feed rate is the most significant factor which affects the vibration as it contributes $59.53 \%$ of total variation, followed by cutting speed (17.53\%) and drill bit type (16.27\%). Minimum value of vibration is observed to be $0.0276 \mu \mathrm{m}$ at $50 \mathrm{~m} / \mathrm{min}, 0.025 \mathrm{~mm} / \mathrm{rev}$ and with TiAlN drill bit type.

Increasing cutting speed by $66.6 \%$ results in good surface roughness by $12.44 \%$ of total variation. From the experimental data, it is observed that $75 \%$ increase in feed rate increases vibration by $18.60 \%$ of total variation. Feed rate contributes $54.50 \%$ to the total variation of vibration, followed by cutting speed (38.95) and drill bit type (5.30\%).

\subsection{SEM Images}

SEM images of machined test specimen are given in Fig. 5. In Fig. 5a fiber pullout is seen. Fig. 5b shows fiber breakage. Fig. 5c shows a sample surface with internal delamination. Fig. 5d shows voids while machining with HSS drill bit at low cutting speed and high feed rate. Fig. 5e shows a rough surface texture that occurred at machining of $30 \mathrm{~m} / \mathrm{min}$ and feed rate 

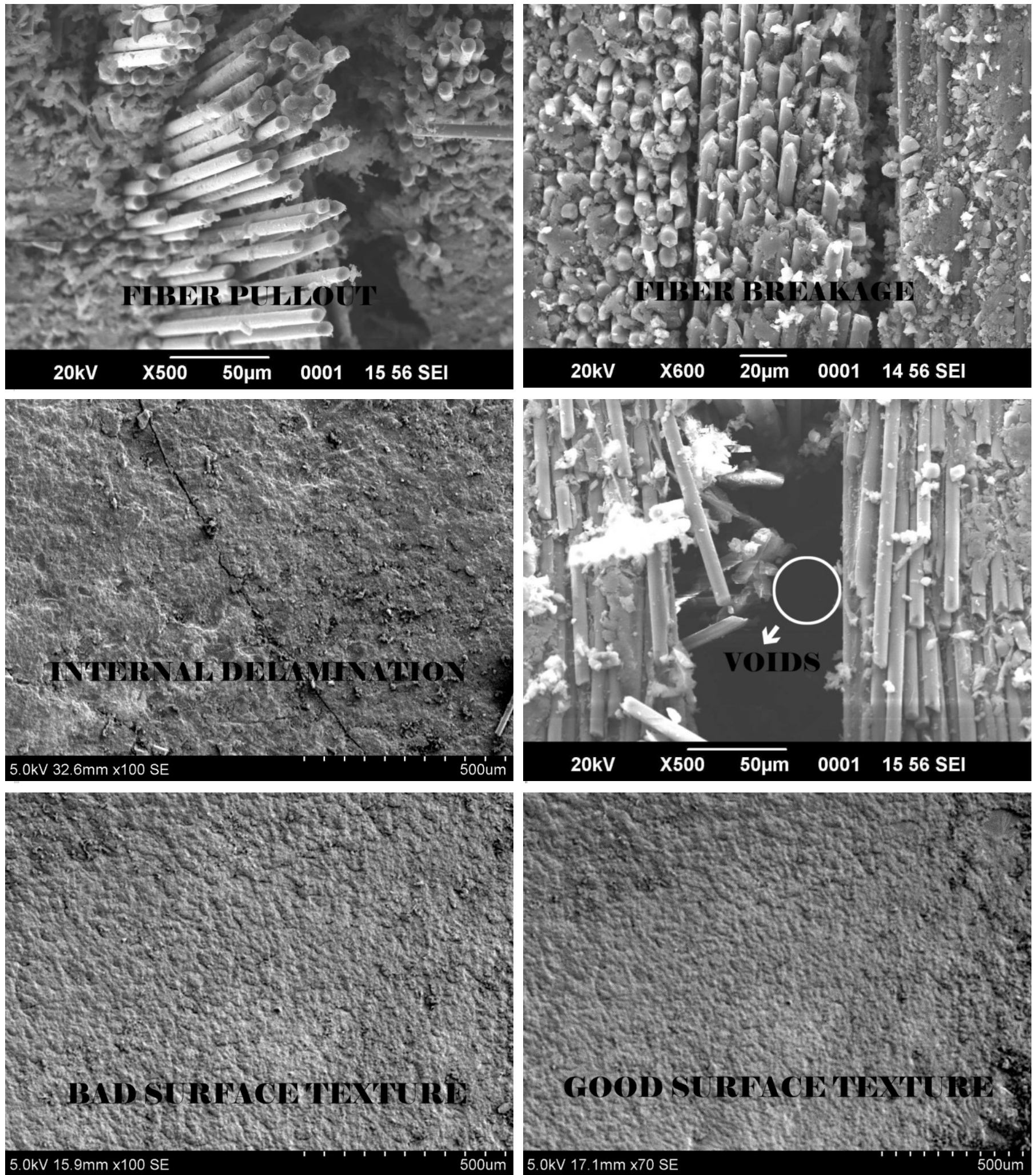

Fig. 5. SEM Images of the test specimen

of $0.1 \mathrm{~mm} / \mathrm{rev}$. Fig. $5 \mathrm{f}$ shows an apparently accep Table surface texture that occurred with TiAlN drill bit tool at $50 \mathrm{~m} / \mathrm{min}$ cutting speed and lowest feed rate employed, $0.025 \mathrm{~mm} / \mathrm{rev}$. The surface quality investigation using SEM suggest optimum surface finish with highest cutting speed employed, lowest feed rate used and hardest tool material TiAlN.

\section{Conclusions}

In this paper, drilling experiments are carried out as per Taguchi $\mathrm{L}_{27}\left(3^{13}\right)$ orthogonal array experimental design, on CFRP laminates using HSS, TiAIN and TiN drill bit type. The machining process parameters are optimized using multi-objective 
1812

Taguchi technique and TOPSIS. The conclusions drawn from the experimental results are summarized as follows:

1. From the multi-objective Taguchi technique, the most predominant process parameter was found to be feed rate, followed by cutting speed and drill bit type.

2. Minimum surface roughness $\left(R_{a}=4.05 \mu \mathrm{m}\right)$, minimum thrust force $\left(F_{o}=54 \mathrm{~N}\right)$, minimum torque $\left(T_{o}=22 \mathrm{Nm}\right)$ and minimum vibration $(0.0276 \mu \mathrm{m})$ was obtained from the optimum combination of process parameters $\left(v_{3} f_{1} d_{2}\right)$.

3. The results of TOPSIS technique are in good agreement with the multi-objective Taguchi technique.

4. The actual values of thrust force, torque, vibration and surface roughness matches closely with those predicted by the second order mathematical model, shows the goodness of the developed models.

5. From the results of ANOVA feed rate is found to be the most predominant factor which affects the responses.

\section{REFERENCES}

[1] S. Arul, L. Vijayaraghavan, S.K. Malhotra, R. Krishnamurthy, Int. .J Mach. Tool Manu. 46 (3), 252-259 (2006).

[2] S. Ramesh, R. Viswanathan, S. Ambika, S, Measurement 78 (1), 63-72 (2016).

[3] M.A. Herbert, N. Shetty, R. Shetty, D.S. Shetty, G.S. Vijay, Appl. Soft. Comput. 41 (1), 466-478 (2016).
[4] M. Kurt, E. Bagci, Y. Kaynak, The Int. J. Adv. Manu.f Tech. 40 (5-6), 458-469 (2009).

[5] T.J. Grilo, R.M.F. Paulo, C.R.M. Silva, J.P. Davim, Compos. Part B-Eng. 45 (1), 1344-1350 (2013).

[6] C.C. Tsao, H. Hocheng, J. Mater. Process. Tech. 203 (1), 342-348 (2008).

[7] S. Sheth, P.M. George, Precis. Eng. 2016.

[8] M. Nouari, G. List, F. Girot, D. Coupard, Wear 255 (7), 1359-1368 (2003).

[9] I.S. Shyha, D.K. Aspinwall, S.L. Soo, S. Bradley, Int. J. Mach. Tool Manu. 49 (12), 1008-1014 (2009).

[10] S. Neseli, Adv. Mech. Eng. 6, 925382 (2014).

[11] A.M. Abrao, P.E. Faria, J.C. Rubio, P. Reis, J.P. Davim, J. Mater. Process Tech. 186 (1), 1-7 (2007).

[12] S. Jayabal, U. Natarajan, The Int. J. Adv. Manuf. Tech. 51 (1-4), 371-381 (2010).

[13] S. Dewangan, S. Gangopadhyay, C.K. Biswas, Measurement 63(1), 364-376 (2015).

[14] A. Gok, Measurement 70 (1), 100-109 (2015).

[15] V. Madhavan, G. Lipczynski, B. Lane, E. Whitenton, J. Manuf. Process. 20 (2), 431-442 (2015).

[16] X. Zhang, S. Yu, Y. Gong, Y. Li, Adv. Mech. Eng. 8 (2), (2016).

[17] R. Bobbili, V. Madhu, A.K. Gogia, Defence Tech. 11 (4), 344-349 (2015).

[18] R.K. Pandey, S.S. Panda, Measurement 59 (1), 9-13 (2015). 